\title{
ANATOMICAL PROTOCOL FOR GAIT ANALYSIS: JOINT KINEMATICS MEASUREMENT AND ITS REPEATABILITY
}

\author{
Magdalena Żuk, Marta Trzeciak \\ Wroclaw University of Technology, Department of Biomedical Engineering, Mechatronics and Theory of Mechanisms, \\ Wrockaw, Poland; e-mail: magdalena.zuk@pwr.edu.pl
}

\begin{abstract}
International Society of Biomechanics has proposed a general reporting standard for joint kinematics based on anatomical reference frames. Nevertheless, the gait analysis protocols based on this standard are still poorly reported. The purpose of the current study is to propose and preliminarily assess the potential of an anatomically based ISB 6-DOF protocol, which combines the ISB reporting standard together with a marker cluster technique. The proposed technical marker set enables full description of the lower limb kinematics (including three-dimensional ankle-foot complex rotations) according to the current biomechanical recommendation. The marker set provides a clinically acceptable inter-trial repeatability and minimal equipment requirements.
\end{abstract}

Keywords: joint kinematics, gait analysis protocol, repeatability, anatomical protocol, marker-set, motion capture system

\section{Introduction}

-Three-dimensional kinematic measures of the human gait provide useful data for clinical practice and biomechanical research (Baker, 2006; Syczewska et al., 2012). Increasingly, quantitative description of the human movement is used as input data in dynamic simulation of the musculoskeletal system (Delp et al., 2007), including joint moment identification using inverse dynamic methods as well as muscle force estimation using optimization based methods (Erdemir et al., 2007; Żuk and Pezowicz, 2016). Furthermore, such data may be helpful in the design of walking machines, exoskeletons (Oliński et al., 2015), limb prosthesis or active orthoses (Dollar and Herr, 2008).

Contemporary quantitative analysis of gait incorporates advanced, still expensive motion capture systems for tracking marker location. The marker set together with the related biomechanical model for mathematical description of lower limb kinematics is called the gait analysis protocol.

The widely used protocol in clinical gait analysis is Conventional Gait Model (Davis et al., 1991; Kadaba et al., 1990) which is better standardised and validated than other models (Baker, 2006); therefore, it seems to be the most appropriate model in clinical practice at the moment. In this protocol, markers are placed both above bony landmarks and wand, therefore, this protocol is not fully anatomical. Simultaneously, the Conventional Gait Model is inconsistent with the ISB reporting standard.

Protocols based on the current ISB recommendation (Wu et al., 2002) are still poorly reported. A recent study evaluated the performance of anatomically based protocols (Manca et al., 2010; Leardini et al., 2007; Ferrati et al., 2008), including those using marker clusters (Collins et al., 2009). However, both those protocols are not fully consistent with the ISB recommendation.

Gait kinematics measured using an anatomically based protocol, which also enables tracking of each segment independently, could increase the accuracy of musculoskeletal modelling and also seems to be more appropriate for consideration of orthoses and exoskeletons design. 
The purpose of the current study is to propose and assess the protocol, which fulfils ISB standard, as well as to present reference data for normal subjects obtained using the proposed protocol. The proposed anatomically based protocol combines the general reporting standard recommended by the International Society of Biomechanics (ISB) together with a marker cluster technique. In the previous paper (Żuk and Pezowicz, 2015), the proposed methodology was presented and comparative analysis with a conventional protocol was conducted on the limited group as a preliminary verification of applied methods. In the current study, the reference data for normal subjects have been collected and inter-trial reproducibility has been validated. Furthermore, the applied methodology have been described in greater detail.

\section{Methods}

Lower limb motion was tracked using a motion capture system (Optotrak Certus, NDI, Canada) with one position sensor, equipped with three embedded infrared cameras (Fig. 1). The system tracked position and orientation of clusters of active markers.
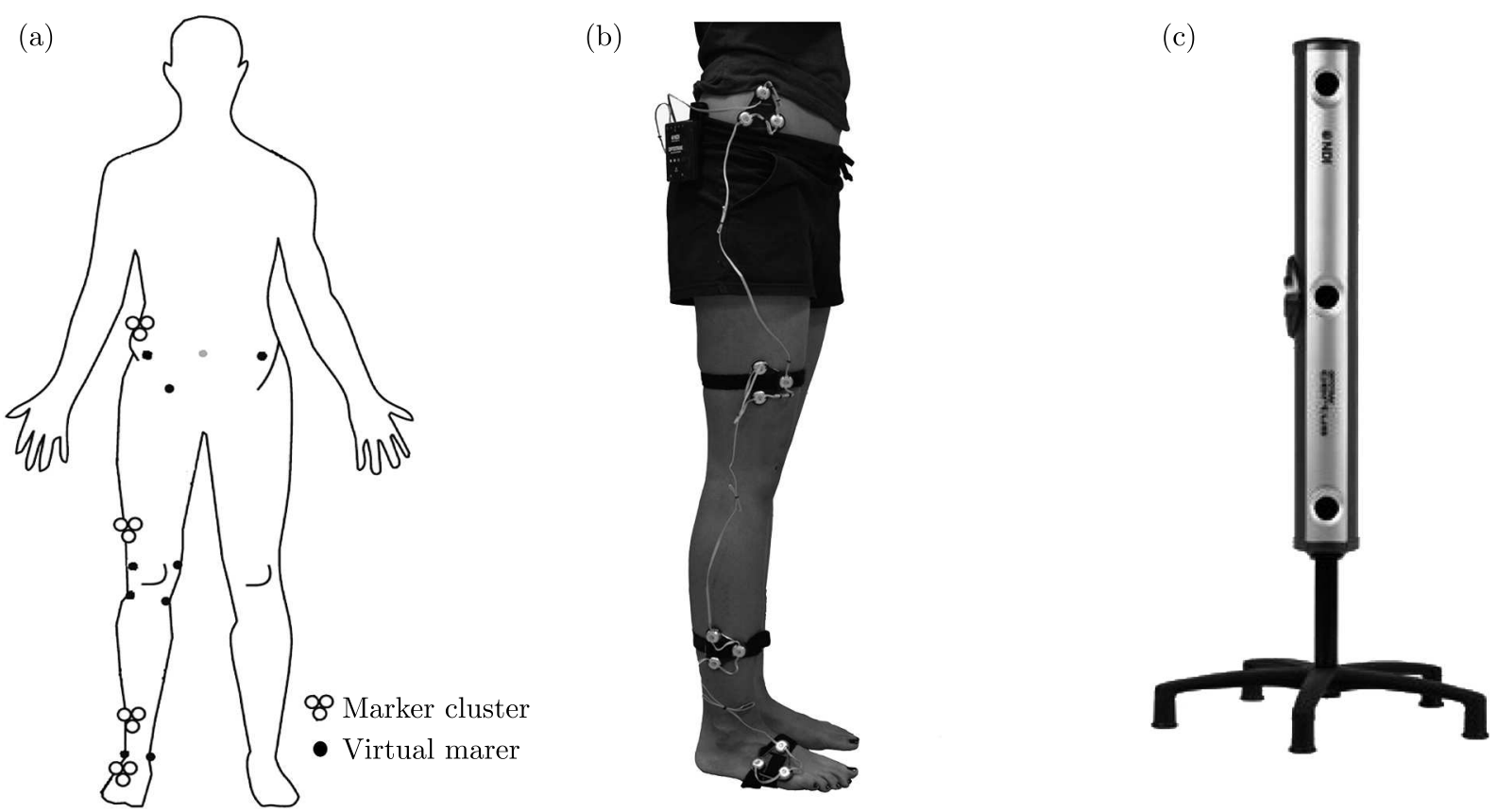

Fig. 1. (a) Marker set including technical markers and virtual markers, (b) marker placement,

(c) motion capture system

Four clusters of active markers were located on pelvis and right lower limb segments: thigh, shank, and foot. The clusters were placed laterally on the distal part of each segment. Each cluster, consisting of three active markers (infrared LEDs) attached on a rigid base (Optotrak Smart Marker Rigid Body, NDI, Canada), was mounted with an adhesive tape and a band. The pelvis cluster was mounted using only adhesive tape. Locations of marker clusters and virtual markers are shown in Fig. 1. Furthermore, two additional virtual markers (on the heel and the metatarsal head) were included for foot visualization and gait phase identification. Davis's regression equation was applied to determine the hip joint centre (Davis et al., 1991)

Anatomical landmarks were defined as virtual markers whose positions with respect to the technical markers (cluster) were measured using a tracked pointer during a static trial. The virtual marker set was designed on the basis of the current ISB recommendation (Wu et al., 2002) for anatomical reference frames. Anatomical coordinate systems of each anatomical segment were defined in pursuance of the paper by Wu et al. (2002) (Fig. 2). 
Pelvis coordinate system

Thigh coordinate system

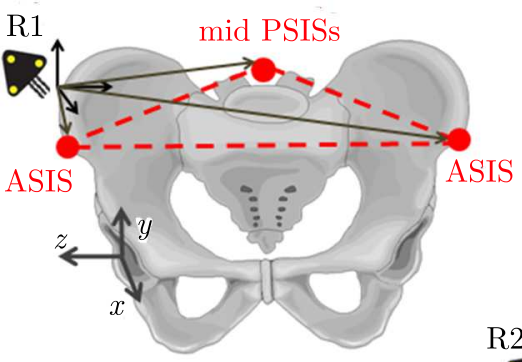

Cluster of markers

- Analomical landmark (virtual marker)

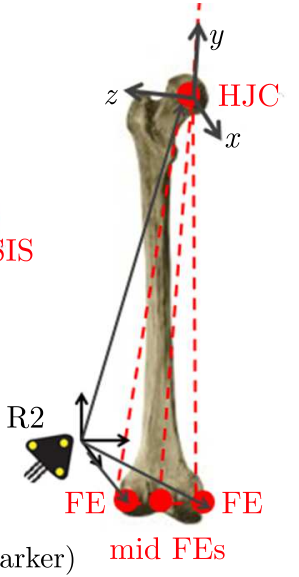

Tibiafibula coordinate system

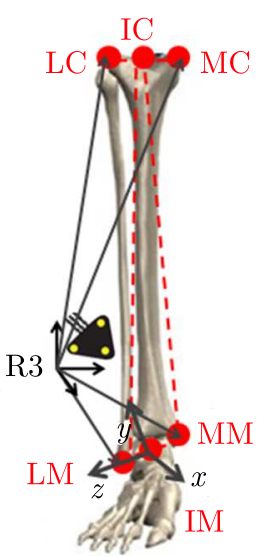

Foot coordinate system

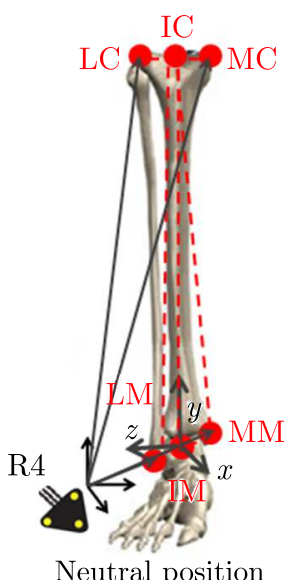

Fig. 2. Anatomical coordinate systems definition according to ISB recommendation (Wu et al., 2002) based on following virual markeres: ASIS - anterior superior iliac spine, midPSIS - midpoint between posterior superior iliac spines, HJC - hip joint centre, FE - femur epicondyle, midFEs - midpoint between femur epicondyles, LC - the most lateral point on the boarder of the lateral tibial condyle, $\mathrm{MC}$ - the most medial point on the border of the medial tibial condyle, IC - the inter-condylar point located modway between the MC and LC, LM - tip of medial malleolus, MM - tip of the medial malleolus

Cardan's angular convention was used to describe relative orientation of adjacent segments (Tupling and Pierrynowski, 1987; Kadaba et al., 1990). In this convention, the joint rotations $\mathbf{R}$ are described as compound rotations

$$
\begin{aligned}
\mathbf{R} & =\left[\begin{array}{lll}
r_{11} & r_{12} & r_{13} \\
r_{21} & r_{22} & r_{23} \\
r_{31} & r_{32} & r_{33}
\end{array}\right]=\mathbf{R}_{Z \gamma} \mathbf{R}_{X \alpha} \mathbf{R}_{Y \beta} \\
& =\left[\begin{array}{ccc}
\cos \gamma & -\sin \gamma & 0 \\
\sin \gamma & \cos \gamma & 0 \\
0 & 0 & 1
\end{array}\right]\left[\begin{array}{ccc}
1 & 0 & 0 \\
0 & \cos \alpha & -\sin \alpha \\
0 & \sin \alpha & \cos \alpha
\end{array}\right]\left[\begin{array}{ccc}
\cos \beta & 0 & \sin \beta \\
0 & 1 & 0 \\
-\sin \beta & 0 & \cos \beta
\end{array}\right] \\
& =\left[\begin{array}{ccc}
\cos \gamma \cos \beta-\sin \gamma \sin \alpha \sin \beta & -\sin \gamma \cos \alpha & \cos \gamma \sin \beta+\sin \gamma \sin \alpha \cos \beta \\
\sin \gamma \cos \beta+\cos \gamma \sin \alpha \sin \beta & \cos \gamma \cos \alpha & \sin \gamma \sin \beta-\cos \gamma \sin \alpha \cos \beta \\
-\cos \alpha \sin \beta & \sin \alpha & \cos \alpha \cos \beta
\end{array}\right]
\end{aligned}
$$

where $\mathbf{R}_{Z \gamma}, \mathbf{R}_{X \alpha}, \mathbf{R}_{Y \beta}$ are rotation matrices corresponding to rotations around anatomical axes, respectively: rotation by an angle $\gamma$ around the frontal axis $Z$, rotation by an angle $\alpha$ around the sagittal axis $X$ and rotation by an angle $\beta$ around the longituidal axis $Y ; r_{i j}$ are rotation matrix elements. Graphical interpretation of the adopted rotation sequence is presented in Fig. 3.

According to the adopted joint angle definition, if $\mathbf{T}_{A L C S 1 \rightarrow A L C S 2}$ is the matrix of transformation from the proximal segment coordinate system to the distal segment coordinate system, which can be like this

$$
\mathbf{T}_{A L C S 1 \rightarrow A L C S 2}=\left[\begin{array}{cccc}
r_{11} & r_{12} & r_{13} & T_{X} \\
r_{21} & r_{22} & r_{23} & T_{Y} \\
r_{31} & r_{32} & r_{33} & T_{Z} \\
0 & 0 & 0 & 1
\end{array}\right]
$$

where $T_{X}, T_{Y}, T_{Z}$ refers to translations, then the anatomical joint angles can be calculated as follows

$$
\alpha=\arcsin r_{32} \quad \beta=\arcsin \frac{-r_{31}}{\cos \alpha} \quad \gamma=\arcsin \frac{-r_{12}}{\cos \alpha}
$$




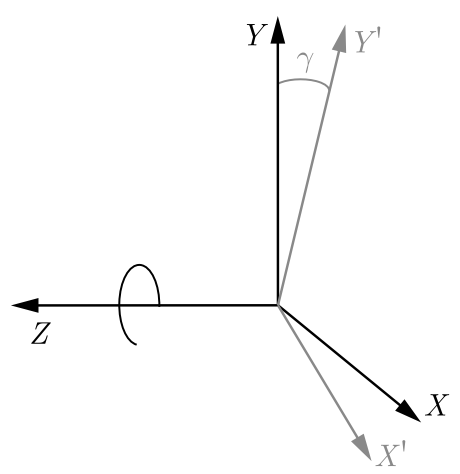

Rotation around axis $Z$ by an angle $\gamma$

Cardan angles $Z-X^{\prime}-Y^{\prime \prime}$ sequence

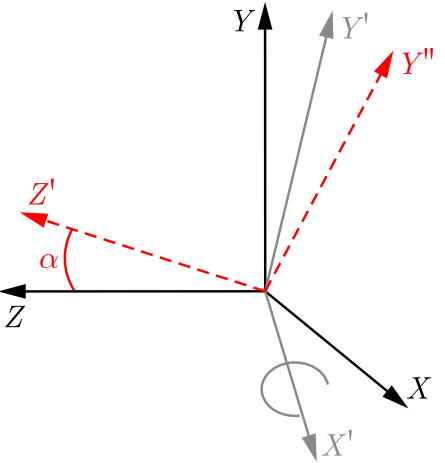

Rotation around axis $X^{\prime}$ by an angle $\alpha$

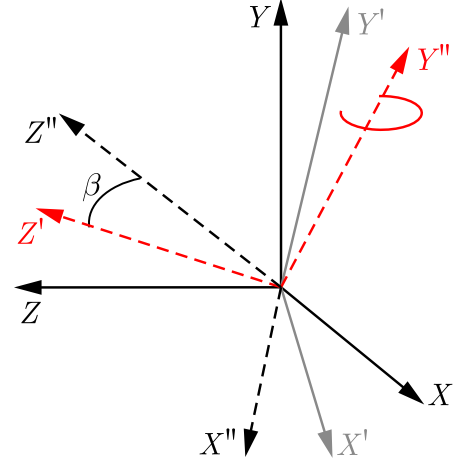

Rotation around axis $Y^{\prime \prime}$ by an angle $\beta$

Fig. 3. Graphical representation of Cardan angle convention

where $\alpha$ is abduction/adduction joint angle, $\beta$ is external/internal rotation angle and $\gamma$ is flexion/extension joint angle.

Data acquisition and joint angle calculation were performed using custom-made software. Data processing, including gait cycle normalisation and smoothing, was performed using Matlab.

Ten able-bodied subjects without walking disability (five females and five males) were analysed (aged $22 \pm 2$ years, weight $66 \pm 11 \mathrm{~kg}$, height $1.75 \pm 0.11 \mathrm{~m}$ ). In the case of experimental methods or repeatability analysis, it was used to combine females and males while preserving the age range, which was shown in the paper by McGinley et al. (2009).

All participants provided written informed consent before participation. The subjects walked barefoot at a preferred pace and three gait cycles were selected.

The mean value and the standard deviation of 12 rotations were calculated over three trials for each sample of the gait cycle in ten subjects. Angle curves were plotted for a single representative subject (mean of three cycles) and for ten subjects (averaged across mean curves of each subject). Inter-trial variability was calculated according to the recommended method (Schwartz et al., 2004; McGinley et al., 2009) and plotted. Average inter-trial variability (AIT) was compared to the corresponding values from recent papers (Manca et al., 2010; Schwartz et al., 2004). Averaged intra-protocol variability was defined as a mean standard deviation over all subjects averaged across the gait cycle.

\section{Results}

Calculated joint rotations (Fig. 4) are related to corresponding data derived from similar biomechanical models (Benedetti et al., 1998; Leardini et al., 2007; Collins et al., 2009). The lowest consistency of the range of motion (ROM) is observed for the ankle angle, for which the anatomical frame definition and the marker set differ considerably from other models. Average inter-trial variability is low (Table 1) and similar to the corresponding data from other studies (Manca et al., 2010; Schwartz et al., 2004). The most repeatable rotation within the subject is pelvis obliquity $\left(0.9^{\circ}\right)$, while the lowest reproducibility is observed for hip internal/external rotation and pelvis rotation $\left(2.6^{\circ}\right)$. The latter results from slight changes of the gait direction during the study. Inter-trial repeatability clearly depends on the phase of gait (Fig. 5). In particular, for knee flexion/extension and ankle inversion/eversion, inter-trial variability doubles during swing phase. Intra-protocol (Table 1) variability is highest for hip flexion/extension $\left(14.2^{\circ}\right)$ and pelvic tilt $\left(12.7^{\circ}\right)$ while for the other angles it does not exceed $10^{\circ}$. 

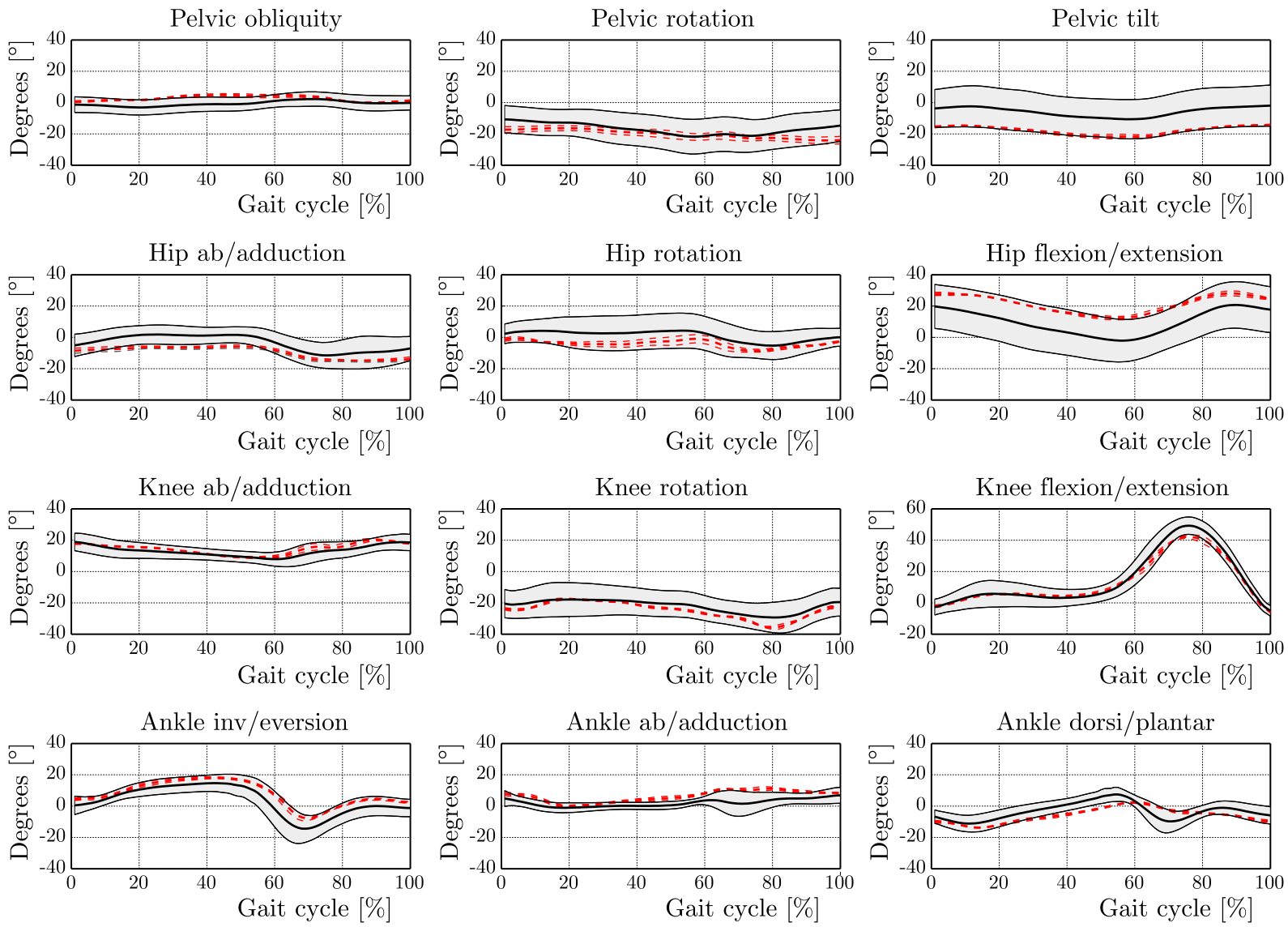

Fig. 4. Kinematic variables as calculated by the ISB 6-DOF of one representative subject (mean across three cycles - gray dashed line, +/- SD - gray dashed thin line) and ten subjects (averaged across mean curves of subjects - black solid line, +/- SD grey band)

Table 1. Average inter-trial and intra-protocol variability over the gait cycle across four subjects. Corresponding values from Manca et al. (2010) and Schwartz et al.(2004)

\begin{tabular}{|l|c|c|c|c|}
\hline \multirow{2}{*}{ Rotations $\left.{ }^{\circ}\right]$} & \multicolumn{3}{|c|}{ Inter-trial } & Intra-protocol \\
\cline { 2 - 5 } & Present study & Manca et al. & Schwartz et al. & Present study \\
\hline \hline Pelvis tilt & 1.2 & 0.9 & $0.8^{*}$ & 12.7 \\
\hline Pelvis obliquity & 0.9 & 1.4 & $0.5^{*}$ & 4.6 \\
\hline Pelvis rotation & 2.6 & 1.7 & $1.0^{*}$ & 10.0 \\
\hline Hip flex/ext & 1.6 & 1.8 & $1.2^{*}$ & 14.2 \\
\hline Hip abd/add & 1.4 & 1.7 & $0.5^{*}$ & 7.1 \\
\hline Hip intr/extr & 2.6 & 2.9 & $1.2^{*}$ & 9.4 \\
\hline Knee flex/ext & 1.9 & 2.2 & 1.6 & 6.3 \\
\hline Knee var/valg & 1.0 & 1.6 & $0.5^{*}$ & 4.8 \\
\hline Knee intr/extr & 1.2 & 4.3 & $1.2^{*}$ & 9.2 \\
\hline Ankle dor/pla & 1.6 & 2.0 & $1.3^{*}$ & 4.5 \\
\hline Ankle inv/ev & 1.8 & 2.3 & - & 6.3 \\
\hline Ankle abd/add & 1.1 & 2.8 & 1.7 & 3.7 \\
\hline
\end{tabular}

* data estimated from figures provide 

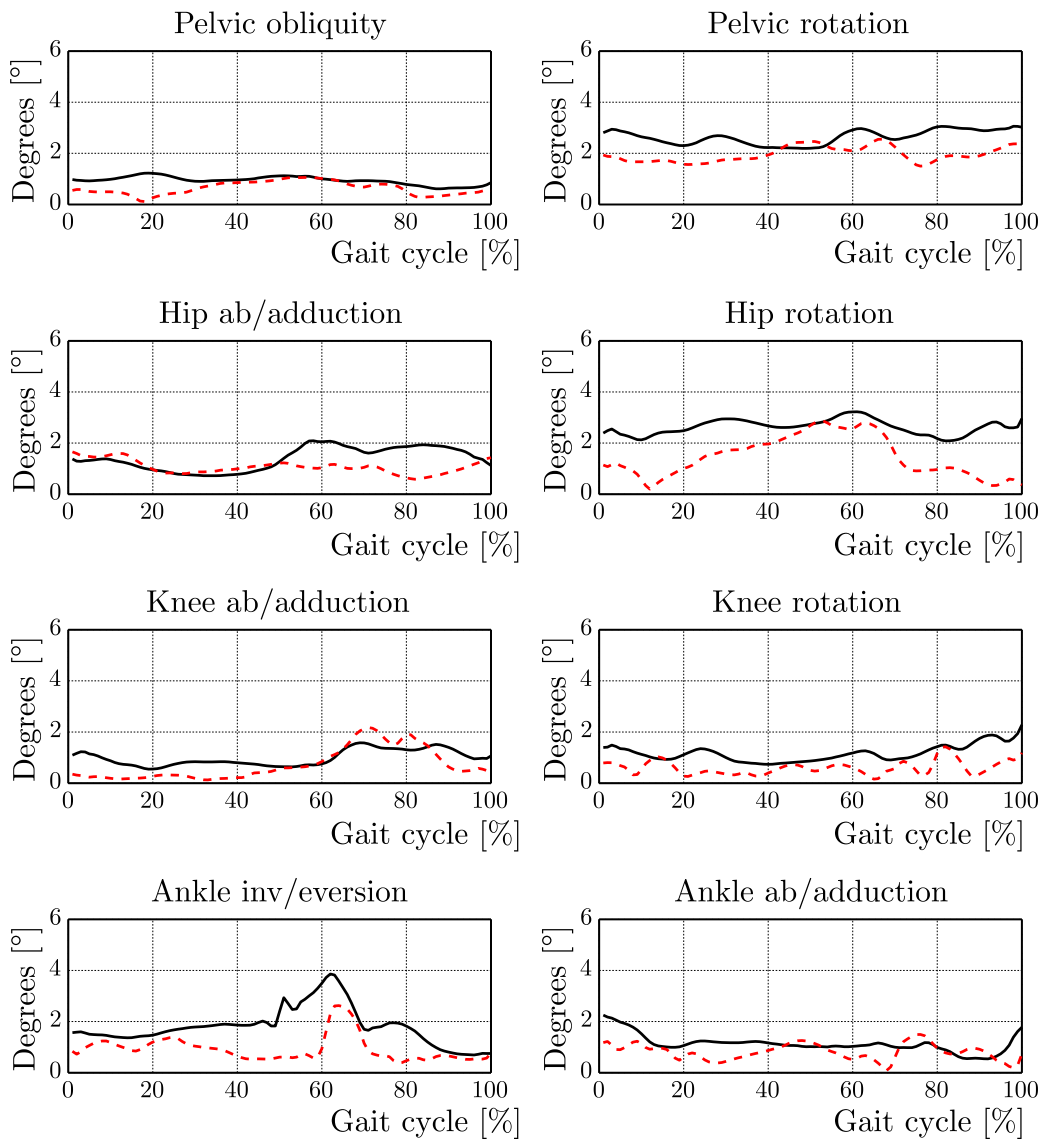
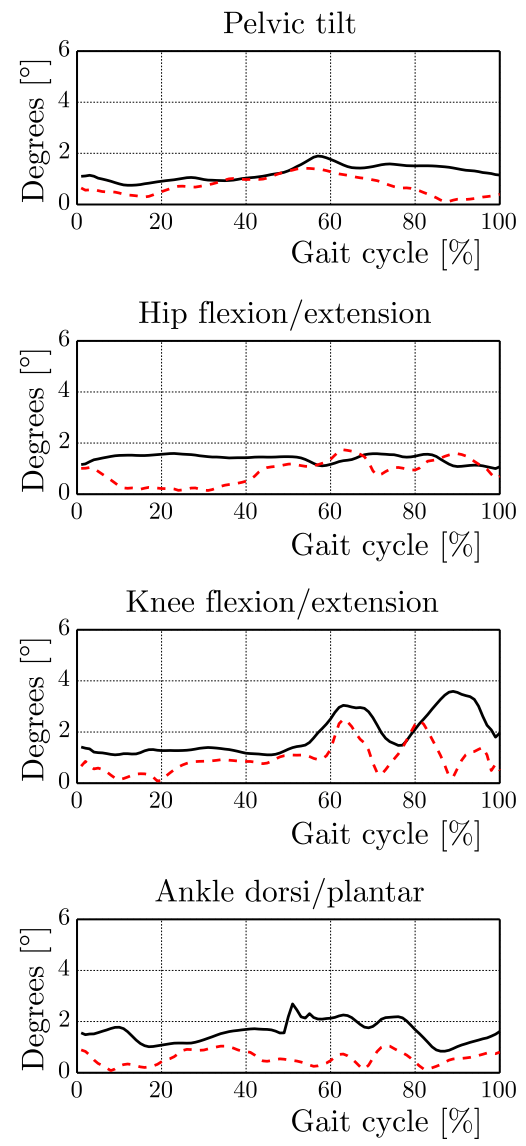

Fig. 5. Patterns of standard deviation across all samples of the gait cycle, one representative subject (gray dashed line) and average for ten subjects (black solid line)

\section{Discussion}

The proposed technical marker set enables full description of lower limb kinematics, including three-dimensional (3D) ankle-foot complex rotations according to the current biomechanical convention (Wu et al., 2002). Lower limb segments are tracked separately without an assumption being made about joint constraints. Thus, this marker set can be applied to determination the joint centres and axes of rotation using functional methods, which was previously reported by Żuk et al. (2014). Besides, marker clusters in combination with an anatomical calibration allow definition of an unlimited number of virtual markers, freely placed within the segment, including those located beyond the "line of sight" of the position sensor. Only one position sensor (consisting of at least two cameras) is needed to track a selected lower limb (clusters located laterally) as well as both limbs (clusters placed frontally). The application of an additional position sensor allows such an arrangement of the clusters, particularly location of the pelvis cluster on the sacrum (Borhani et al., 2013), which could reduce soft tissue artefacts (STA).

Reference data for normal subjects have been collected. Although the obtained selected joint angle curves are in agreement with the literature (Leardini et al., 2007; Collins et al., 2009; Benedetti et al., 1998), caution is recommended when comparing the results among different protocols, especially in the case of non-sagittal planes (Ferrati et al., 2008).

The obtained average inter-trial variability is acceptable in clinical application according to previous papers by Schwartz et al. (2004) and McGinley et al. (2009). A relatively low intertrial variability indicates proper mounting of marker clusters, which eliminares sliding during examination. Further evaluation of the ISB 6-DOF protocol should include analysis of inter- 
session and inter-assessor repeatability. However, inter-session and inter-assessor repeatability appear to be close to those achieved with other anatomically based protocols (Manca et al., 2010) due to a similar source of variability (palpation of external bony landmarks).

An anatomically based protocol in which virtual markers are placed on bony landmarks without wands, increase reliability of musculoskeletal modelling by more accurate matching of marker trajectories to the scaled model.

The ISB 6-DOF protocol provides a full 3D description of lower limb kinematics according to the current recommendation ( $\mathrm{Wu}$ et al., 2002) with acceptable inter-trial variability. There are some limitations of the proposed method. The use of only one position sensor is associated with sub-optimal pelvis cluster location, which can affect pelvis and hip rotations. Moreover, at the present time, lack of relevant reference data for patients restricts the use of these methods in clinical practice.

Nevertheless, the proposed marker set can minimize the required equipment and, thereby, can enhance the availability of gait analysis in research and clinical applications.

\section{References}

1. BAKER R., 2006, Gait analysis methods in rehabilitation, Journal of Neuro Engineering and Rehabilitation, $\mathbf{3}$

2. Benedetti M.G., Catani F., Leardini A., Pignotti E., GianniniG., 1998, Data management in gait analysis for clinical applications, Clinical Biomechanics, 13, 3, 204-215

3. Borhani M., McGregor A.H., Bull A.M.J., 2013, An alternative technical marker set for the pelvis is more repeatable than standard pelvic marker set, Gait and Posture, 38, 4, 1032-1037

4. Collins T.D., Ghoussayni S.N., Ewins D.J., Kent J.A., 2009, A six degrees-of-freedom marker set for gait analysis: Repeatability and comparison with modified Helen Hayes set, Gait and Posture, 30, 173-180

5. Davis III R.B., Õunpuu S., Tyburski D. Gage J.R., 1991, A gait analysis data collection and reduction technique, Human Movement Science, 10, 5, 575-587

6. Delp S.L, Anderson F.C., Arnold A.S., Loan P., Habib A., John C.T., Guendelman E., TheLen D.G., 2007, OpenSim: open-source software to create and analyze dynamic simulations of movement, IEEE Transactions on Biomedical Engineering, 54, 11, 1940-1950

7. Dollar A.M., Herr H., 2008, Lower extremity exoskeletons and active orthoses: challenges and state-of-the-art, IEEE Transactions on Robotics, 24, 1, 144-158

8. Erdemir A, McLean S, Herzog W., van den Bogert A.J., 2007, Model-based estimation of muscle forces excerted during movements, Clinical Biomechanics, 22, 131-154

9. Ferrati A., Benedetti M.G., Pavan E., Frigo C., Bettinelli D., Rabuffetti M., CrenNa P., Leardini A., 2008, Quantitative comparsion of five current protocols in gait analysis, Gait and Posture, 28, 207-216

10. Kadaba M.P., Ramakrishnan H., Wootten M., 1990, Measurement of lower extremity kinematics during level walking, Journal of Orthopedic Research, 8, 383-393

11. Leardini A., Sawacha Z., Paolini G., Ingrosso S., Nativo R., Benedetti M.G., 2007, A new anatomically based protocol for gait analysis in children, Gait and Posture, 26, 560-571

12. Manca M., Leardini A., Cavazza S., Ferraresi G., Marchi P., Zanaga E., Benedetti M.G., 2010, Repeatability of new protocol for gait analysis in adult subject, Gait and Posture, 32, $282-284$

13. McGinley J.L., Baker R., Wolfe R., Morris M.E., 2009, The reliability of three dimensional kinematic gait measurements: a systematic review, Gait and Posture, 29, 360-369 
14. Oliński M., Lewandowski B., Gronowicz A., 2015, Type synthesis and preliminary design of devices supporting lower limb's rehabilitation, Acta of Bioengineering and Biomechanics, 17, 1, 117-127

15. Schwartz M.H., Trost J.P., Wervey R.A., 2004, Measurement and management of errors in quantitative gait data, Gait and Posture, 20, 196-203

16. Syczewska M., Graff K., Kalinowska M., Szczerbik E., Domaniecki J., 2012, Influence of the structural deformity of the spine on the gait pathology in scoliotic patients, Gait and Posture, 35, 2, 209-213

17. Tupling S.J., Pierrynowski M.R., 1987, Use of cardan angles to locate rigid bodies in threedimensional space, Medical and Biological Engineering and Computing, 25, 527-532

18. Wu G., Siegler S., Allard P., Kirtley C., Leardini A., Rosenbaum D., Whittle M., D’Lima D.D., Cristofolini L., Witte H., Schmid O., Stokes I., 2002, ISB reccommendation on definitions of joint coordinate system of various joints for reporting of human joint motion part I: ankle, hip, and spine, Journal of Biomechanics, 35, 543-548

19. Żuk M., Pezowicz C., 2015, Kinematic analysis of a six-degrees-of-freedom model based on ISB recommendation: a repeatability analysis and comparison with conventional gait model, Applied Bionics and Biomechanics, Article ID 503713, 1-9

20. Żuk M., Pezowicz C., 2016, The influence of uncertainty in body segment mass on calculated joint moments and muscle forces, Information Technologies in Biomedicine, 5, 349-359

21. Żuk M., Świątek-Najwer E., Pezowicz C., 2014, Hip joint centre localization: evaluation of formal methods and effects on joint kinematic, Information Technologies in Biomedicine, 4, 57-67 